\title{
Variations on a unifying theme
}

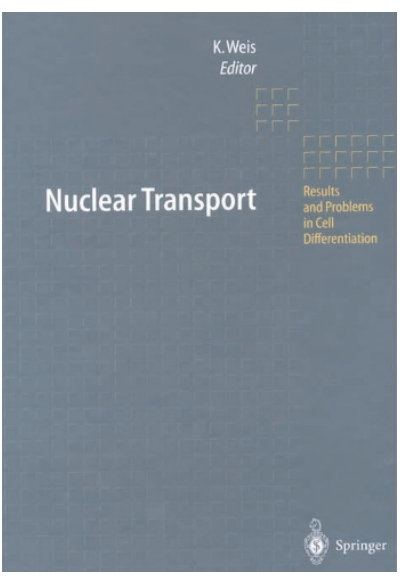

\author{
Nuclear Transport \\ edited by K. Weis \\ Springer-Verlag $\cdot 2002$ \\ Hardback, $£ 72.50 / \$ 110$ \\ Valérie Doye
}

A defining feature of eukaryotic cells is the presence of intracellular membranes that delineate specific compartments, of which the most prominent is the nucleus. Segregating the genome from cytosolic processes with the nuclear envelope meant that the cell had to develop efficient processes to transport proteins, RNAs, and large macromolecular complexes such as ribosomes, in and out of the nucleus. That nucleocytoplasmic transport could be tightly regulated allowed eukaryotic cells to finely control a myriad of cellular mechanisms including gene expression, signal transduction, cell cycle and differentiation. Major insights into the nature and character of the essential components of the nuclear transport machinery have been gained over the past 10 years, and although there are numerous reviews on this topic, the field is now complex enough to justify a whole textbook. In Nuclear Transport, edited by Karsten Weis, the various aspects of nuclear transport are covered in nine chapters, written by well-known investigators. Each of these reviews is clearly written, and most of the authors do not over-emphasize their own research, but instead provide a complete overview of the pertinent questions being addressed within a given area. Because each of the chapters can be read independently, this leads to some overlap - but in ways this is welcome, as it allows different points of view on specific aspects of nuclear transport to be re-emphasized.

Anchored in the nuclear envelope, the nuclear pore complexes (NPCs) control all exchanges between the cytoplasm and nucleus. The first two chapters review the structure, composition and function of these highly symmetric and dynamic assemblies in yeast (Strambio-de-Castillia and Rout) and vertebrates (Fahrenkrog and Aebi). Although identification of yeast nuclear pore constituents, termed nucleoporins, was close to completion in 2000, identifying vertebrate nucleoporins is still in progress. So we will have to wait for future reviews to be able to effectively compare the yeast NPCs with their more elaborate vertebrate counterparts. These first two chapters also introduce the reader to the basic mechanisms of nucleocytoplasmic transport, which are further developed in the following sections. The now commonly used in vitro nuclear import assay was a major breakthrough for this field, which led to the characterization of the importin/karyopherin- $\beta$ family of transport receptors and the Ran GTPase. As emphasized in most chapters, this abundant GTPase is predominantly found in its GTP-bound form in the nucleus, and in its GDP-bound form in the cytoplasm. This Ran gradient accounts for the directionality of all karyopherin (that is, both importin and exportin)mediated transport processes, and is tightly regulated, as detailed in another chapter by Bishoff, Scheffzek and Pongstingl. As the first transport pathway to be characterized, analysis of the so-called classical NLSmediated protein import pathway has reached an advanced stage, in which crystal structures of several of its components are being resolved. Elena Conti describes how these static images can be combined to better understand the molecular basis of directionality and specificity of nuclearimport processes.

Unfortunately, the diversity of nuclear import pathways, which involve a variety of importin- $\beta$-like transport factors, as well as more specific mechanisms, is not stressed in this volume. But the importance of these alternative pathways, which several contributors expected to be fully covered in an accompanying chapter, shines through the many discussions focused on the specific affinities that different FG (phenylalanine-glycine)-repeat-containing nucleoporins have for the various karyopherin-cargo complexes. In contrast, the diversity of nuclear export pathways is thoroughly covered by reviews focused on the export of protein and ribonucleoprotein, transfer RNA, and messenger RNA, and a specific chapter by Bryan Cullen strengthens the contribution of retroviruses as model systems for studying nuclear export. Fornerod and Ohno clearly highlight the predominant involvement of exportin-1/CRM1 in most (but not all) protein and ribonucleoprotein export pathways. But there are several examples of nuclear export that do not fit the exportinmediated pathways. In particular, although CRM1 is involved in the export of a few specific RNA molecules, including incompletely spliced viral mRNAs, Elisa Izaurralde describes the various proteins specifically implicated in the export of most cellular mRNAs. Similarly, although tRNA export involves a specific exportin, the chapter by Simos, Großhans and Hurt indicates that aminoacylation could be involved in an alternative tRNA export pathway. These two reviews also show how intimately coupled mRNA and tRNA export are with the transcription, splicing, and translation machineries. A final chapter by Schüller and Ruis illustrates some of the strategies used to regulate nucleocytoplasmic transport.

I would certainly recommend this book to both newcomers and established scientists in the field. However, the lack of a chapter devoted to the diversity of nuclear-import pathways and the sparsely distributed figures render this volume less accessible to undergraduate students. In contrast to many other books, most of the chapters in this edition are reasonably upto-date, as they include references up to the end of 2000. Because each chapter contains numerous citations, this volume will be an extremely useful reference in the next few years. By including perspectives about the ongoing research and discussing the main open questions in the field, this book might also stimulate young scientists to join this exciting and highly dynamic field.

Valérie Doye is in the UMR144 CNRS Institut Curie, Research Division 26, rue d'Ulm, 75248 Paris cedex 05, France. e-mail:vdoye@curie.fr 